\title{
DIE 7. ARBEITSTAGUNG DER DEUTSCHEN GESELLSCHAFT FÜR KARTOGRAPHIE IN FREIBURG IM BREISGAU UND IN ZÜRICH \\ EDUARD IMHOF
}

Vom 25. bis 28. September 1957 führte die Deutsche Gesellschaft für Kartographie in den Räumen der Universität zu Freiburg i. Br. eine von nahezu 300 Teilnehmern besuchte und durch Herrn Dipl.-Ing. Veit, Präsident des Bayerischen Landesvermessungsamtes, meisterhaft geleitete Arbeitstagung durch.

Anläßlich der Eröffnungssitzung sprach Prof. Dr. h. c. Ed. Iмноғ (Zürich) über «Naturalistik und Abstraktion in der kartographischen Geländedarstellung». Er leitete damit ein in das zur Diskussion gestellte Hauptthema der Tagung, zu dem sich in weiteren Referaten auch Dr. Fritz Hölzel (Rheda), Hannes KreuzKAMP (Freiburg i. Br.) und Dr. R. Wewer (Heidelberg) äußerten. Es handelte sich vor allem um Fragen einer unmittelbar anschaulichen, naturähnlichen und morphologisch charakteristischen Geländedarstellung durch Farb- und Schattentöne in Karten großer, wie auch kleiner Maßstäbe, um Neuerungen mechanisierter Kartenerstellung und um reproduktionstechnische Probleme. Eine Kartenausstellung in der Freiburger Universität war ebenfalls der Geländedarstellung gewidmet.

Weiter referierten Prof. Dr. R. Oenme (Karlsruhe) über die Geschichte der Kartographie des südlichen Schwarzwaldes und der angrenzenden Oberrheinebene, und Vermessungsrat Dipl.-Ing. J. SснӥNke (Karlsruhe) über topographische Geländeaufnahmen.

Das Gebiet der thematischen Karte wurde durch Prof. Dr. ERIch Otremba (Hamburg) aufgegriffen durch einen grundlegenden Vortrag über wirtschaftsgeographische Karten. Der Redner wies hierbei eindrücklich auf die Schwierigkeiten dieser inhaltlich und formalgraphisch meist sehr komplexen Karten hin, auf Schwierigkeiten, die oft Trug- und Vexierbilder entstehen lassen, wenn die Hand des Kartengraphikers nicht durch gründliches geographisches und methodisches Wissen geführt wird.

An Stelle des leider zu früh verstorbenen Herrn Kurt MaIr (Stuttgart) wurde an der Generalversammlung Herr Dr. Theodor Siewke (Frankfurt a. M.) zum neuen Präsidenten der Gesellschaft gewählt.

Eine wunderschöne Fahrt mit Autocars, von Prof. Dr. Friedrich Metz (Freiburg i. Br.) kulturgeographisch ausgezeichnet kommentiert, brachte am 28. September etwa 220 Kongreßteilnehmer über die Höhen des Schwarzwaldes nach Schaffhausen, an den Rheinfall und nach Zürich zu einem Besuch des Kartographischen Instituts der Eidg. Technischen Hochschule. Hier referierte Prof. ImHoF über einige grundsätzliche Fragen der Kartographie. Anschließend besichtigten die Besucher die Ateliers des Institutes und eine Karten- und Reliefausstellung, die in einigen Räumen der Hochschule eingerichtet worden war.

Nach einer Stadtrundfahrt reisten die deutschen Gäste gegen Abend über Basel wieder nach Freiburg zurück.

Eingehendere Berichterstattungen über diese Tagung werden in den «Kartographischen Nachrichten», Jahrgang 1958 (Verlag Velhagen \& Klasing, Bielefeld) erscheinen.

\section{ERINNERUNG AN NATIONALRAT ERNST BÄRTSCHI}

Am 29. Juli dieses Jahres feierte Nationalrat und alt Stadtpräsident von Bern, Dr. ERnst BärTsCHı seinen 75. Geburtstag. Die außerordentlichen Verdienste, die der Jubilar um Ansehen und heutige Bedeutung der Geographie als Lehrfach an den schweizerischen Mittelschulen besitzt, rechtfertigen bei diesem Anlaß einige Worte der Anerkennung.

Es ist den meisten heute im Schuldienst wirkenden Kollegen kaum mehr bewußt, welcher Anstrengungen es bedurfte, bis die Geographie als Mittelschulfach die Stellung erreichte, die ihr 